\title{
A HIGHER ORDER INVARIANT OF DIFFERENTIAL MANIFOLDS
}

\author{
GREGORY A. FREDRICKS, PETER B. GILKEY AND PHILLIP E. PARKER \\ Dedicated to Aldo Andreotti on the 65th anniversary of his birth and \\ Paul Olum on his retirement from the University of Oregon
}

ABstract. We discuss conditions under which a lens space is $s$ th order flat.

\section{INTRODUCTION}

Let $M$ be a smooth manifold of dimension $m$. We put a Riemannian metric on $M$ and identify the tangent space $T(M)$ with the cotangent space $T^{*}(M)$ henceforth. If $E$ is a vector bundle over $M$, let $J(E)=E \oplus(E \otimes T(M))$ be the jet bundle of $E$; this is the natural setting for studying first order differential equations. Let $J^{s}(E)=J\left(J^{s-1}(E)\right)$ be the iterated jet bundle and let $J^{s}(M)=$ $J^{s}(T(M))=T(M) \otimes(1 \oplus T(M))^{s}$.

$E$ is said to be flat if it admits a connection whose curvature vanishes identically; bundles can admit inequivalent flat structures. $M$ is said to be flat if the tangent bundle $T(M)$ is flat. $M$ is sth order flat if $J^{s}(M)$ admits a flat structure. The set of integers $s$ for which $M$ is $s$ th order flat is an invariant of $M$; the smallest such integer is denoted by $\alpha(M)$ if it exists; otherwise we let $\alpha(M)=\infty$. In this note, we will study the alpha invariant for lens spaces; this is a natural context to phrase the question.

If $J^{s}(M)$ admits a flat structure for some $s$, the rational Pontrjagin classes of $M$ are zero in positive degrees and $T(M)$ is rationally trivial. Let $C P^{k}$ be complex projective space. If $k>1$, then $p_{1}\left(C P^{k}\right) \neq 0$ so $J^{s}\left(C P^{k}\right)$ is never flat and $\alpha\left(C P^{k}\right)=\infty$. The spherical space forms behave quite differently and provide a family of interesting examples for which $\alpha<\infty$. We establish the following notational conventions. $G$ will be a finite group and $\tau: G \rightarrow O(m+1)$ will be a fixed point free representation of $G$. If $G$ admits such a $\tau$, we say $G$ is a spherical space form group. The existence of $\tau$ places restrictions on $G$. In particular, the $p$-Sylow subgroups of $G$ are cyclic if $p$ is odd and either cyclic or quaternion if $p=2$. Let

$$
\mathbf{Z}_{n}=\left\{\lambda \in \mathbf{C}: \lambda^{n}=1\right\} \subset U(1) \subset O(2),
$$

Received by the editors May 2, 1988 and, in revised form, February 7, 1989. Presented January 11,1989 , at the 847th meeting of the AMS in Phoenix.

1980 Mathematics Subject Classification (1985 Revision). Primary 57R25; Secondary 55R50, 53C05, 58A20.

Research partially supported by the NSF and NSA. 


$$
Q_{2}=\{ \pm 1, \pm i, \pm j, \pm k\} \subset S U(2)=S^{3} \subset O(4)
$$

be the cyclic group of order $n$ and the quaternion group of order 8 ; let $\rho_{1}: \mathbf{Z}_{n} \rightarrow O(2)$ and $\tau_{0}: Q_{2} \rightarrow O(4)$ be the natural fixed point free embeddings. We refer to Wolf [11] for a discussion of other examples.

Let $M=M(G, \tau)=S^{m} / \tau(G)$ be the resulting quotient manifold; $M$ is a compact Riemannian manifold which inherits a metric of constant sectional curvature 1 ; all complete manifolds of constant sectional curvature 1 arise in this fashion. These are the spherical space forms and have been classified by Wolf [11]. The classifying space

$$
B G=\lim _{\operatorname{dim}(\tau) \rightarrow \infty} M(G, \tau)
$$

so these spaces are important in algebraic topology. We shall be primarily interested in the case $G$ cyclic; these define the lens spaces. If $G=\{1\}, M$ is the sphere $S^{m}$; if $G=\mathrm{Z}_{2}, M$ is real projective space $R P^{m}$. We summarize below the major results of this paper:

Theorem 0.1. Let $M=M(G, \tau)$ have dimension $m \neq 7$.

(a) $\alpha(M)<\infty . J^{s}(M)$ admits a flat structure if and only if $s \geq \alpha(M)$.

(b) $\alpha\left(S^{m}\right)=0$ if $m=1,3,7 . \alpha\left(S^{m}\right)=1$ otherwise. If $m=1,3$, then $\alpha(M)=0$. Otherwise $\alpha(M) \geq 1$.

(c) Choose s so $(m+1)^{s}<\left|\widetilde{K} O\left(R P^{m}\right)\right| \leq(m+1)^{s+1}$. Then $\alpha\left(R P^{m}\right)=s$.

(d) If $(a, b)=1$, let $G=\mathbf{Z}_{a b}$. Then

$$
\alpha(M)=\max \left\{\alpha\left(M\left(\mathbf{Z}_{a}, \tau\right)\right), \alpha\left(M\left(\mathbf{Z}_{b}, \tau\right)\right)\right\} .
$$

(e) Let $G=\mathbf{Z}_{4}$ or $G=\mathbf{Q}_{2}$ and let $s=\alpha\left(R P^{m}\right)$. Suppose either $s$ is even, or $m \leq 100,000$, or $m$ is large. Then $\alpha(M)=s$.

(f) Let $G=\mathbf{Z}_{n}$ for $n$ an odd prime power. $\exists$ a constant $m_{0}=m_{0}(n)$ so

(i) If $\operatorname{dim}(M)>m_{0}$, then $\alpha(M) \leq n-2$.

(ii) If $m$ is odd and $m>m_{0}, \exists M$ so $\alpha(M)=1$.

(iii) If $M$ is a classical lens space and $\operatorname{dim}(M)>m_{0}$, then $\alpha(M)=n-2$.

This shows sup $\alpha\left(R P^{m}\right)=\infty$ while $\sup _{\tau} \alpha\left(M\left(\mathbf{Z}_{n}, \tau\right)\right)<\infty$ if $n$ is odd; the behavior of $\alpha$ at the prime 2 is very different from the behavior at odd primes as we shall see. As $T(M) \oplus 1$ admits a flat structure, $T(M)$ is stably flat so the computation of $\alpha(M)$ is a delicate one. If $m=1,3$ then $T(M)$ is trivial so $\alpha(M)=0$; if $m \neq 1,3,7$ then $T\left(S^{m}\right)$ is not trivial so $\alpha\left(S^{m}\right) \geq 1$ which implies $\alpha(M) \geq \alpha\left(S^{m}\right) \geq 1$. Since $\operatorname{dim} J^{s}(M)>\operatorname{dim}(M)$ for $s>1$ we will be in the stable range and can use methods from $K$-theory to determine $\alpha(M)$. The case $m=7$ is exceptional and is excluded from consideration for the most part. The proof of (e) relies on a lemma from number theory we have checked by explicit computation for $m \leq 100,000$ and for which we have an asymptotic proof based on the methods of Shorey-Tijdeman; we believe it is true in general but have not checked it owing to the difficulty of making effective the constants which appear. 
In $\S 1$, we will review the basic facts we shall need and prove $0.1(\mathrm{a}, \mathrm{b}, \mathrm{c})$. In $\S 2$, we will prove $0.1(\mathrm{~d})$ which reduces the calculation of $\alpha$ for lens spaces to studying prime powers. In $\S 3$, we study the prime 2 and prove $0.1(\mathrm{e})$. In $\S 4$ we study odd primes and prove $0.1(\mathrm{f})$. In the final section, we will study $\alpha\left(\mathbf{Z}_{31}, k \cdot \rho_{1}\right)$ as a function of $k$ in some detail to illustrate some further phenomena.

\section{THE SPHERES AND PROJECTIVE SPACES}

Let $F \in\{O, U\}$ be either the orthogonal or unitary group. Let $\operatorname{Vect}_{F}^{\nu}(M)$ be the set of isomorphism classes of vector bundles of dimension $\nu$ over $M$ with structure group $F$. Let $\nu \cdot 1$ be the trivial bundle of dimension $\nu$. The map $E \rightarrow E \oplus 1$ defines an isomorphism from $\operatorname{Vect}_{F}^{\nu}(M) \rightarrow \operatorname{Vect}_{F}^{\nu+1}(M)$ for $\nu>m$ if $F=O$ and for $2 \nu \geq m$ if $F=U$; this is called the stable range. We can cancel in the stable range; $E_{0} \oplus E_{1} \cong E_{0} \oplus E_{2}$ implies $E_{1} \cong E_{2}$. Let $K F(M)$ and $\widetilde{K} F(M)$ be the unreduced and reduced $K$-theory groups; we will not need the higher $K$-theory groups for what follows. If $E \in \operatorname{Vect}_{F}^{\nu}(M)$, let $[E] \in K F(M)$;

$$
\gamma: E \rightarrow[E]-\nu \cdot[1]
$$

is an isomorphism from $\operatorname{Vect}_{F}^{\nu}(M)$ to $\widetilde{K} F(M)$ in the stable range. If $E$ is real, let $c(E)=E \otimes C$ be the corresponding complex vector bundle; if $E$ is complex, let $r(E)$ be the underlying real bundle; $c$ and $r$ extend to $K$-theory. $c(r(E))=E \oplus E^{*}$ and $r(c(E))=2 E . \quad c$ is a ring morphism; $r$ is not a ring morphism. Since $r\left(E_{1}\right) \otimes_{\mathbf{R}} r\left(E_{2}\right)=r\left(E_{1} \otimes_{\mathbf{C}}\left(E_{2} \oplus E_{2}^{*}\right)\right)$, image $(r)$ is a subring.

If $M=S^{1}$, then $T(M)=1$ and $J^{s}(M)$ is trivial for all $s$. We assume $m>1$ for the most part. Let $M=M(G, \tau)$. As $S^{m}$ is simply connected, we can identify $G$ with the fundamental group of $M$. If $m=2 k-1$ is odd and if $\tau$ is fixed point free, $\tau$ is conjugate to a representation $\tau: G \rightarrow U(k) \subset O(2 k)$; see Wolf [11]. The only even-dimensional spherical space forms are the spheres $S^{2 k}$ and the projective spaces $R P^{2 k}$.

If $\sigma$ is a representation of $G$, let $\pi(\sigma)$ be the corresponding vector bundle over $M . \pi(\sigma)$ admits a canonical flat structure. Conversely if a bundle $E$ has a flat structure, the holonomy of $E$ defines a representation $\sigma$ of $\pi_{1}(M)=G$ so that $E=\pi(\sigma)$. Let $R F(G)$ be the group representation ring and let $R F_{0}(G)$ be the augmentation ideal. The actual representations form a positive cone in $R F(G)$; we shall say $\sigma_{1} \geq \sigma_{2}$ if $\sigma_{1}-\sigma_{2} \geq 0$; this defines a partial order on $R F(G)$. Extend $[\pi]$ to a ring morphism $[\pi]: R F(G) \rightarrow K F(M)$; the restriction defines $[\pi]: R F_{0}(G) \rightarrow \widetilde{K} F(M)$. Let $K F_{\text {flat }}(M)$ and $\widetilde{K} F_{\text {flat }}(M)$ be the image of $[\pi]$; this is generated by bundles which (stably) admit flat structures.

Lemma 1.1. Let $M=M(G, \tau)$.

(a) $T(M) \oplus 1=\pi(\tau) .\left[J^{s}(M)\right] \in K O_{\text {flat }}(M) \forall s$.

(b) $\left|\widetilde{K} O_{\text {flat }}(M)\right|<\infty$. 
(c) If $s \geq 1$, the following statements are equivalent.

(i) $J^{s}(M)=\pi(\sigma)$,

(ii) $\left[\pi\left(\tau^{s+1}-\tau^{s}-\sigma\right)\right]=0$.

Proof. $T\left(S^{m}\right) \oplus 1$ is isomorphic to the restriction of the tangent bundle of $R^{m+1}$ to $S^{m}$ so $T\left(S^{m}\right) \oplus 1=(m+1) \cdot 1$. This isomorphism is equivariant with respect to the action of $O(m+1)$ and descends to an isomorphism $T(M) \oplus 1=\pi(\tau)$. Consequently $[T(M)] \in K O_{\text {flat }}(M)$; as $K O_{\text {flat }}$ is a ring, $\left[J^{s}(M)\right] \in K O_{\text {flat }}(M)$; this proves (a). If $\sigma \in R O_{0}(G)$, the rational Pontrjagin classes of $\pi(\sigma)$ vanish so $[\pi(\sigma)]$ is a torsion class. Since $R O_{0}(G)$ is a finitely generated $\mathbf{Z}$ module (b) follows. If $s \geq 1$, we are in the stable range so the following statements are equivalent:

(1) $T(M) \otimes(T(M) \oplus 1)^{s}=\pi(\sigma)$,

(2) $T(M) \otimes(T(M) \oplus 1)^{s} \oplus(T(M) \oplus 1)^{s}=\pi(\sigma) \oplus(T(M) \oplus 1)^{s}$,

(3) $(T(M) \oplus 1)^{s+1}=\pi(\sigma) \oplus(T(M) \oplus 1)^{s}$,

(4) $\pi(\tau)^{s+1}=\pi(\sigma) \oplus \pi(\tau)^{s}$.

Let $\widetilde{K} O_{\text {flat }}(M)=\left\{x_{1}, \ldots, x_{\mu}\right\}$. Let $x_{i}=\left[\pi\left(\sigma_{1}(i)\right)\right]-\left[\pi\left(\sigma_{2}(i)\right)\right]$ for $\sigma_{\nu}(i) \geq 0$. Let $a_{i}$ be the order of $\gamma\left\{\pi\left(\sigma_{2}(i)\right)\right\}$ in $\widetilde{K} O(M)$ and let $\sigma_{3}(i)=\sigma_{1}(i)+\left(a_{i}-1\right)$. $\sigma_{2}(i)$. Then $x_{i}=\gamma\left\{\pi\left(\sigma_{3}(i)\right)\right\}$. Let $t=\max \left\{m+1, \operatorname{dim} \sigma_{3}(1), \ldots, \operatorname{dim} \sigma_{3}(\mu)\right\}$ and choose $u$ so $\operatorname{dim} J^{u}(M) \geq t$. Since $\left[J^{u}(M)\right] \in K O_{\text {flat }}(M), \gamma\left\{J^{u}(M)\right\}=x_{j}$ for some $j$. Therefore $\gamma\left\{J^{u}(M)-\pi\left(\sigma_{3}(j)\right)\right\}=0$ so $J^{u}(M)=\pi\left(\sigma_{3}(j)\right)+$ $\left\{\operatorname{dim} J^{u}(M)-\operatorname{dim} \sigma_{3}(j)\right\} \cdot 1$ since we are in the stable range. This shows $J^{u}(M)$ is flat. Choose $s$ minimal so $J^{s}(M)=\pi(\sigma)$ is flat. If $v \geq s$, then $J^{v}(M)=$ $J^{s}(M) \otimes(T(M) \oplus 1)^{v-s}=\pi\left(\sigma \otimes \tau^{v-s}\right)$ so $J^{v}(M)$ is flat. This proves Theorem $0.1(\mathrm{a})$.

Let $H$ be a subgroup of $G$. The pull back of $J^{s}(M(G, \tau))$ by the natural covering projection is $J^{s}(M(H, \tau))$. Consequently $J^{s}(M(G, \tau))$ is flat implies $J^{S}(M(H, \tau))$ is flat. This proves

Lemma 1.2. If $H \subseteq G$, then $\alpha(M(G, \tau)) \geq \alpha(M(H, \tau))$.

We can now prove Theorem $0.1(\mathrm{~b})$. We compute

$$
\left(T\left(S^{m}\right) \oplus 1\right)^{2}=(m+1)^{2} \cdot 1=\left\{T\left(S^{m}\right) \oplus 1\right\} \oplus\left\{(m+1)^{2}-(m+1)\right\} \cdot 1,
$$

so $\alpha\left(S^{m}\right) \leq 1$ by Lemma 1.1. $S^{1}$ is flat as $T\left(S^{1}\right)$ is trivial. Otherwise, $S^{m}$ is simply connected so a bundle over $S^{m}$ has a flat structure if and only if it is trivial. Hence $\alpha\left(S^{m}\right)=0$ when $T\left(S^{m}\right)$ is trivial; this happens if $m \in\{1,3,7\}$ (see Bott-Milnor [1]); otherwise $T\left(S^{m}\right)$ is not trivial and $\alpha\left(S^{m}\right)=1$. If $m=$ $1,3, T(M)$ is trivial so $\alpha(M)=0$. If $m \neq 1,3,7, \alpha(M) \geq \alpha\left(S^{m}\right)=1$. This completes the proof of $0.1(\mathrm{~b})$; our techniques do not suffice to study $\alpha(M)$ if $m=7$.

We compute $\alpha\left(R P^{m}\right)$ to prove Theorem $0.1(\mathrm{c})$. If $s=0$, then $m=1,3$, or 7 and it is well known that $T\left(R P^{m}\right)$ is trivial in these dimensions. Consequently we suppose $m \neq 1,3,7$ henceforth. Let $2^{f(m)}=\left|\tilde{K} O\left(R P^{m}\right)\right|$ be the 
order of the reduced real $K$-theory group;

$$
f(m)=\left\{\begin{array}{ll}
{[m / 2]} & \text { if } m \equiv 6,7,8 \bmod 8 \\
{[m / 2]+1} & \text { otherwise }
\end{array}\right\} .
$$

We list below some values of $f(m)$ and $s$ where $s$ is defined by $(m+1)^{s}<$ $2^{f(m)} \leq(m+1)^{s+1}$.

\section{TABLE 1}

\begin{tabular}{|cccccccccccc|}
\hline$m$ & $f$ & $s$ & $m$ & $f$ & $s$ & $m$ & $f$ & $s$ & $m$ & $f$ & $s$ \\
\hline 1 & 1 & 0 & 9 & 5 & 1 & 17 & 9 & 2 & 25 & 13 & 2 \\
2 & 2 & 1 & 10 & 6 & 1 & 18 & 10 & 2 & 26 & 14 & 2 \\
3 & 2 & 0 & 11 & 6 & 1 & 19 & 10 & 2 & 27 & 14 & 2 \\
4 & 3 & 1 & 12 & 7 & 1 & 20 & 11 & 2 & 28 & 15 & 3 \\
5 & 3 & 1 & 13 & 7 & 1 & 21 & 11 & 2 & 29 & 15 & 3 \\
6 & 3 & 1 & 14 & 7 & 1 & 22 & 11 & 2 & 30 & 15 & 3 \\
7 & 3 & 0 & 15 & 7 & 1 & 23 & 11 & 2 & 31 & 15 & 2 \\
8 & 4 & 1 & 16 & 8 & 1 & 24 & 12 & 2 & 32 & 16 & 3 \\
\hline
\end{tabular}

Let $\rho_{1}$ be the nontrivial representation of $\mathrm{Z}_{2} ; \pi\left(\rho_{1}\right)=L$ is the classifying bundle over $R P^{m}$. Let $x=[1]-[L]$. Then $x$ has order $2^{f(m)}$ and generates $\widetilde{K} O\left(R P^{m}\right)$. Let $a, b, c, d$ be nonnegative integers with $a+b=c+d>m$. The following statements are equivalent:

(i) $a \cdot 1+b \cdot L=c \cdot 1+d \cdot L$,

(ii) $a \equiv c \bmod 2^{f(m)}$,

(iii) $b \equiv d \bmod 2^{f(m)}$.

We wish to find $a, b$ so $J^{s}\left(R P^{m}\right)=a \cdot 1+b \cdot L$; this is equivalent by Lemma 1.1 to $\left(T\left(R P^{m}\right) \oplus 1\right)^{s+1}=a \cdot 1+b \cdot L+\left(T\left(R P^{m}\right) \oplus 1\right)^{s}$. Since $T\left(R P^{m}\right) \oplus 1=(m+1) \cdot L$, this means

$$
(m+1)^{s+1} \equiv\left\{\begin{array}{ll}
b & \text { if } s \text { is even } \\
a & \text { if } s \text { is odd }
\end{array}\right\} \bmod 2^{f(m)} .
$$

We show $\alpha\left(R P^{m}\right) \leq s$ by finding $(a, b)$. Define

$$
(a, b)=\left\{\begin{array}{ll}
\left(2^{f(m)}-(m+1)^{s},(m+1)^{s+1}-2^{f(m)}\right) & \text { if } s \text { is even } \\
\left((m+1)^{s+1}-2^{f(m)}, 2^{f(m)}-(m+1)^{s}\right) & \text { if } s \text { is odd }
\end{array}\right\} .
$$

Conversely suppose $t \geq 1$ and $J^{t}\left(R P^{m}\right)=c \cdot 1+d \cdot L$. Then

$$
(m+1)^{t+1} \equiv\left\{\begin{array}{ll}
d & \text { if } s \text { is even } \\
c & \text { if } s \text { is odd }
\end{array}\right\} \bmod 2^{f(m)} .
$$

As $c$ and $d$ are less than $(m+1)^{t+1}, 2^{f(m)} \leq(m+1)^{t+1}$. As $(m+1)^{s}<$ $2^{f(m)} \leq(m+1)^{s+1}, s \leq t$. This completes the proof of Theorem $0.1(\mathrm{c})$. The 
cases $s$-even and $s$-odd are completely symmetric for $Z_{2}$; this will not be the case when we study $\mathbf{Z}_{4}$ and $Q_{2}$ in $\S 3$.

Table 1 shows $\alpha$ is not monotonic at $m=31$.

Theorem 1.3. Let $2^{p-1} \equiv 1 \bmod p, m=2^{p}-1$, and $\nu=\left(2^{p-1}-1\right) / p$. Then $\alpha\left(R P^{m}\right)=\nu-1$ and $\alpha\left(R P^{m-1}\right)=\alpha\left(R P^{m+1}\right)=\nu$.

Remark. If $p$ is prime, then $2^{p-1} \equiv 1 \bmod p$ by Fermat's theorem. There are also composite numbers for which this is true.

Proof. We need to establish the following estimates:

(i) $\left(2^{p}+1\right)^{\nu}<2^{\nu p+1}$

(ii) $2^{\nu p+1} \leq\left(2^{p}+1\right)^{\nu+1}$,

(iii) $\left(2^{p}-1\right)^{\nu}<2^{\nu p}$,

(iv) $2^{\nu p} \leq\left(2^{p}-1\right)^{\nu+1}$.

Set $n=2^{p}$. We prove (i) by computing:

$$
\begin{gathered}
\nu=\left(2^{p-1}-1\right) / p<2^{p} /(2 p)<n / 2 \\
(1+1 / n)^{\nu}<(1+1 / n)^{n / 2}<e^{1 / 2}<2 \\
\left(2^{p}+1\right)^{\nu}<2 \cdot 2^{p \nu} .
\end{gathered}
$$

(ii) and (iii) are immediate. Finally let $x=2^{-p} \in(0,1 / 8]$. Then

$$
\ln (1-x) / x=-\left(1+x / 2+x^{2} / 3+\cdots\right) \geq-1 /(1-x) \geq-8 / 7
$$

so $(1-x)^{1 / x} \geq 1 / 6$. Since $\nu \leq 2^{p},\left(1-2^{-p}\right)^{\nu} \geq(1-x)^{1 / x} \geq 1 / 6$ and

$$
\left(2^{p}-1\right)^{\nu}\left(2^{p}-1\right) \geq 6^{-1} \cdot\left(2^{p}-1\right) \cdot 2^{\nu p} \geq 2^{\nu p} \text {. }
$$

Note that $m \equiv 7 \bmod 8$ as $p \geq 3$, so $f(m)=[m / 2]=2^{p-1}-1=p \nu$. Because $2^{f(m)}=2^{p \nu}=(m+1)^{\nu}, \alpha\left(R P^{m}\right)=\nu-1$. Because $m+1 \equiv 0 \bmod 8$, $f(m+1)=f(m)+1=p \nu+1$. Because $\left(2^{p}+1\right)^{\nu}<2^{\nu p+1} \leq\left(2^{p}+1\right)^{\nu+1}$, $\alpha\left(R P^{m+1}\right)=\nu$. Because $m-1 \equiv 6 \bmod 8, f(m-1)=f(m)=\nu p$. Because $\left(2^{p}-1\right)^{\nu}<2^{\nu p} \leq\left(2^{p}-1\right)^{\nu+1}, \alpha\left(R P^{m-1}\right)=\nu$.

\section{Separating out the PRIMES}

The only even-dimensional spherical space forms are the spheres and real projective spaces which were studied in $\S 1$; we assume henceforth $m$ is odd. Any fixed point free representation $\tau$ is conjugate in $O(2 k=m+1)$ to a complex representation so $M=M(G, \tau)$ admits a stable complex structure.

\section{Lemma 2.1.}

(a) $\widetilde{K} U(M)=\widetilde{K} U_{\text {flat }}(M)=R U_{0}(G) / \operatorname{det}(I-\tau) \cdot R U(G)$.

(b) If $|G|$ is even, then $\widetilde{K} O(M)=\widetilde{K} O_{\text {flat }}(M)$.

(c) If $|G|$ is odd, then $\widetilde{K} O(M)=\widetilde{K} O_{\mathrm{flat}}(M) \oplus \widetilde{K} O\left(S^{2 k-1}\right)$ and $c$ is injective on $\widetilde{K} O_{\text {flat }}(M)$.

Remark. $\widetilde{K} O\left(S^{2 k-1}\right)=0$ or $\mathrm{Z}_{2}$ depending on the parity of $k$. 
Proof. This is well known; see for example Gilkey [3, Theorem 3.6] and GilkeyKaroubi [5, Theorem 1].

For the remainder of this section let $n=a \cdot b$ where $a$ and $b$ are coprime. If $\nu$ divides $n$, let $M_{\nu}=M\left(\mathbf{Z}_{\nu}, \tau\right)$, and let $M=M_{n}$. Let $\rho_{s}(\lambda)=\lambda^{s}: \mathbf{Z}_{n} \rightarrow U(1)$ where $s$ is defined $\bmod n$. $\rho_{s}$ is fixed point free if and only if $(s, n)=1$. Let $\operatorname{irr}_{F}(G)$ be the set of irreducible representations up to linear equivalence.

$$
\begin{gathered}
\operatorname{irr}_{U}\left(\mathbf{Z}_{n}\right)=\left\{\rho_{s}\right\}_{0 \leq s<n} . \\
R U\left(\mathbf{Z}_{n}\right)=\mathbf{Z} \cdot \rho_{0} \oplus \cdots \oplus \mathbf{Z} \cdot \rho_{n-1}=\mathbf{Z}\left[\rho_{1}\right] /\left(\left(\rho_{1}\right)^{n}=1\right) . \\
\operatorname{irr}_{O}\left(\mathbf{Z}_{n}\right)=\left\{\begin{array}{ll}
\left\{\rho_{0}, r\left(\rho_{1}\right), \ldots, r\left(\rho_{(n-1) / 2}\right)\right\} & \text { if } n \text { is odd } \\
\left\{\rho_{0}, \rho_{n / 2}, r\left(\rho_{1}\right), \ldots, r\left(\rho_{(n-2) / 2}\right)\right\} & \text { if } n \text { is even }
\end{array}\right\} .
\end{gathered}
$$

Lemma 2.2.

(a) $|\widetilde{K} U(M)|=n^{k}$.

(b) Let $\varepsilon=1$ if $n$ is odd and $\varepsilon=2$ if $n$ is even.

(i) $\left|\widetilde{K} O_{\text {flat }}(M)\right|=\varepsilon^{j+1} \cdot n^{j}$ if $k=2 j+1$ and $m \equiv 1 \bmod 4$.

(ii) $\left|\widetilde{K} O_{\text {flat }}(M)\right|=\varepsilon^{j+1} \cdot n^{j-1}$ if $k=2 j$ and $m \equiv 3 \bmod 8$.

(iii) $\left|\widetilde{K} O_{\mathrm{flat}}(M)\right|=\varepsilon^{j} \cdot n^{j-1}$ if $k=2 j$ and $m \equiv 7 \bmod 8$.

(c) Pullback defines isomorphisms $\widetilde{K} F_{\text {flat }}(M) \simeq \widetilde{K} F_{\text {flat }}\left(M_{a}\right) \oplus \widetilde{K} F_{\text {flat }}\left(M_{b}\right)$.

Proof. See Gilkey [4, Lemma 4.2 and Theorem 0.4] for (a, b); (c) follows from $(\mathrm{a}, \mathrm{b})$ and from Lemma 2.1 .

If $\nu$ divides $n$, let $i_{\nu}^{*}: R F\left(\mathbf{Z}_{n}\right) \rightarrow R F\left(\mathbf{Z}_{\nu}\right) \rightarrow 0$ be the natural restriction.

\section{Lemma 2.3.}

(a) $i_{a}^{*} \oplus i_{b}^{*}$ is surjective from $R U_{0}\left(\mathbf{Z}_{n}\right)$ to $R U_{0}\left(\mathbf{Z}_{a}\right) \oplus R U_{0}\left(\mathbf{Z}_{b}\right)$.

(b) Let $n=2 c$ and let $k \geq 2$. Then $\left[\pi\left(\rho_{c}\right) \oplus \pi\left(\rho_{0}\right)\right] \notin r\{K U(M)\}$.

(c) If $J^{s}(M)=\pi(\sigma)$ for $s \geq 1$, then $\sigma \in r\left\{R U\left(\mathbf{Z}_{n}\right)\right\}$.

Proof. Let $\sigma_{\nu}=\sum_{i}\left(\rho_{\nu(i)}-\rho_{0}\right) \in R U_{0}\left(\mathbf{Z}_{\nu}\right)$ for $\nu=a, b$; we set $\nu(i)=0$ for $i$ large. Choose $n(i)$ so $n(i) \equiv a(i) \bmod a$ and $n(i) \equiv b(i) \bmod b$ and let $\sigma_{n}=\sum_{i}\left(\rho_{n(i)}-\rho_{0}\right)$. This proves (a) since $i_{\nu}^{*}\left(\sigma_{n}\right)=\sigma_{\nu}$ for $\nu=a, b$; we note (a) is false if we replace $R U_{0}$ by $R O_{0}$; if $(a, b, c)=(2,3,6)$, there is no $\sigma \in R O_{0}\left(\mathbf{Z}_{6}\right)$ so $i_{2}^{*}(\sigma)=\rho_{1}-\rho_{0}$ and $i_{3}^{*}(\sigma)=r\left(\rho_{1}\right)-2 \cdot \rho_{0}$. The first Chern class is a map from $K U\left(M_{n}\right)$ to $H^{2}\left(M_{n} ; \mathbf{Z}\right) \cong \mathbf{Z}_{n}$ which satisfies $c_{1}\left(\pi\left(\rho_{s}\right)\right)=s$. The first Chern class of the complexification of a real bundle is zero. Since $c_{1}\left(\pi\left(\rho_{c}+\rho_{0}\right)\right) \neq 0,\left[\pi\left(\rho_{c}+\rho_{0}\right)\right]$ is not the underlying real virtual bundle of a complex virtual bundle; this proves (b). Since $s \geq 1, \operatorname{dim}\left(J^{s}(M)\right)=m \cdot(m+1)^{s}$ is even. If $n$ is odd, then there is only one real representation of $Z_{n}$ so the coefficient $x_{0}$ of $\rho_{0}$ in $\sigma$ is even and $\sigma=\left(x_{0} / 2\right) \cdot r\left(\rho_{0}\right)+\sum_{1 \leq i \leq(n-1) / 2} x_{i} \cdot r\left(\rho_{i}\right)$. If $n=2 c$ is even, there are two real representations of $Z_{n}$. Let $\sigma=x_{0} \cdot \rho_{0}+x_{c} \cdot$ $\rho_{c}+\sum_{1 \leq i<c} x_{i} \cdot r\left(\rho_{i}\right)$; we must show $x_{0}$ and $x_{c}$ are even. Suppose the contrary; since $\operatorname{dim}(\sigma)$ is even, both $x_{0}$ and $x_{c}$ are odd so $\sigma=\rho_{0}+\rho_{c}+r\left(\sigma^{\prime}\right)$. Since 
image $(r)$ is a subring and $T(M) \oplus 1 \in \operatorname{image}(r)$,

$$
\left[J^{s}(M)\right]=\left[(T(M) \oplus 1)^{s+1}\right]-\left[(T(M) \oplus 1)^{s}\right] \in \operatorname{image}(r) .
$$

This implies $\left[\pi\left(\rho_{0}\right) \oplus \pi\left(\rho_{c}\right)\right] \in$ image $(r)$ which is false.

Let $\alpha_{0}=\max \left\{\alpha\left(M_{a}\right), \alpha\left(M_{b}\right)\right\}$. By Lemma 1.2, $\alpha_{0} \leq \alpha(M)$. Conversely, suppose $s \geq \alpha_{0}$. Choose $\sigma_{\nu}$ so $J^{s}\left(M_{\nu}\right)=\pi\left(r\left(\sigma_{\nu}\right)\right)$ for $\nu=a, b$. Then $J^{s}(M)=\pi\left(r\left(\sigma_{n}\right)\right)$; this completes the proof of Theorem 0.1(d).

\section{The AlPha INVARIANT OF $S^{m} / Q_{2}$ AND $S^{m} / \mathbf{Z}_{4}$}

In this section, we will prove Theorem $0.1(\mathrm{e})$. We suppose $m \neq 1,3,7$ for the remainder of this section to avoid low-dimensional pathologies. We recall the following result from Shorey-Tijdeman [9]; see also Turk [10].

Theorem 3.1 (Shorey-Tijdeman). Let $\delta<1$. Let $x, y, a, b$ be positive integers so $x^{a}<y^{b} . \exists b_{0}=b_{0}(\delta, x)$ so if $b \geq b_{0}$, then $x^{a}+x^{\delta a}<y^{b}$.

Corollary 3.2. Let $m \leq 100,000$ or let $m$ be large. Let $s=\alpha\left(R P^{m}\right)$ be odd.

(a) If $m=8 \nu+7$, then $2^{4 \nu+2}+3 \cdot 2^{2 \nu+2} \leq(m+1)^{s+1}$.

(b) If $m=4 \nu+1$, then $2^{2 \nu+1}+2^{\nu+1} \leq(m+1)^{s+1}$.

Proof. We checked the cases $1 \leq m \leq 100,000$ numerically. Let $m$ be large and let $(x, y, a, b)=(2,8 \nu+8,4 \nu+3, s+1)$ or $(2,4 \nu+2,2 \nu+1, s+1)$ so $x^{a} \leq y^{b}$. Since $s$ is odd, $2^{4 \nu+3} \neq(8 \nu+8)^{s+1}$; similarly $(4 \nu+2)$ is not a power of 2 so $2^{2 \nu+1} \neq(4 \nu+2)^{s+1}$. This shows $x^{a}<y^{b}$. Since $2^{f(m)} \leq$ $(m+1)^{s+1},[m / 2] \ln (2) / \ln (m+1) \leq s+1$ so $\lim _{m \rightarrow \infty} s(m)=\infty$. By Theorem $3.1 x^{a}+x^{\delta a}<y^{b}$ if $m$ is large.

Let $Q_{2}=\{ \pm 1, \pm i, \pm j, \pm k\}$. The natural inclusion $\tau: Q_{2} \subset S U(2)=S^{3}$ defines a fixed point free representation; any fixed point free representation of $Q_{2}$ is a multiple of $\tau$. There are 4 linear representations of $Q_{2}$ defined by

$$
\begin{array}{llll}
\rho_{0}(i)=1, & \rho_{i}(i)=1, & \rho_{j}(i)=-1, & \rho_{k}(i)=-1, \\
\rho_{0}(j)=1, & \rho_{i}(j)=-1, & \rho_{j}(j)=1, & \rho_{k}(j)=-1 ;
\end{array}
$$

$\operatorname{irr}\left(Q_{2}\right)=\left\{\rho_{0}, \rho_{i}, \rho_{j}, \rho_{k}, \tau\right\}$. Let $M=M\left(Q_{2}, k \cdot \tau\right)$ have dimension $m=$ $4 k-1$. Let $u=\rho_{i}+\rho_{j}+\rho_{k}$ and let $x(b, c)=\left[\pi\left\{b \cdot\left(u-3 \rho_{0}\right)+c \cdot\left(r(\tau)-4 \rho_{0}\right)\right\}\right] \in$ $\widetilde{K} O(M)$.

\section{Lemma 3.3.}

(a) If $m=8 \nu+3$, then $x(b, c)=0$ if and only if $b \equiv 0 \bmod 2^{2 \nu}$ and $c \equiv 0 \bmod 2^{4 \nu}$.

(b) If $m=8 \nu+7$, then $x(b, c)=0$ if and only if $b \equiv 0 \bmod 2^{2 \nu}$ and $c-2^{2 \nu+1} \cdot b \equiv 0 \bmod 2^{4 \nu+3}$.

Proof. We use the computation of $\tilde{K} O(M)$ from Fujii [2, Theorem 1.3]. Set

$$
\begin{array}{ll}
\alpha_{0}=\left[\pi\left\{\rho_{i}-\rho_{0}\right\}\right], & \beta_{0}=\left[\pi\left\{\rho_{j}-\rho_{0}\right\}\right], \\
\delta_{0}=\left[\pi\left\{r(\tau)-4 \rho_{0}\right\}\right], & x_{0}=\left[\pi\left\{5 \rho_{0}+\rho_{i}+\rho_{j}+\rho_{k}-2 \cdot r(\tau)\right\}\right] .
\end{array}
$$


Then $x(b, c)=b \cdot x_{0}+(c+2 b) \cdot \delta_{0}$ and

$$
\begin{gathered}
\tilde{K} O(M)=\left\{\begin{array}{ll}
\mathbf{Z}_{2^{2 \nu+2}} \oplus \mathbf{Z}_{2^{2 \nu+2}} \oplus \mathbf{Z}_{2^{4 \nu}} \oplus \mathbf{Z}_{2^{2 \nu}} & \text { if } m=8 \nu+3 \\
\mathbf{Z}_{2^{2 \nu+2}} \oplus \mathbf{Z}_{2^{2 \nu+2}} \oplus \mathbf{Z}_{2^{4 \nu+3}} \oplus \mathbf{Z}_{2^{2 \nu}} & \text { if } m=8 \nu+7
\end{array}\right\}, \\
\text { with generators }=\left\{\begin{array}{ll}
\left\{\alpha_{0}, \beta_{0}, \delta_{0}, x_{0}+2 \cdot \delta_{0}\right\} & \text { if } m=8 \nu+3 \\
\left\{\alpha_{0}, \beta_{0}, \delta_{0}, x_{0}+\left(2+2^{2 \nu+1}\right) \delta_{0}\right\} & \text { if } m=8 \nu+7
\end{array}\right\} .
\end{gathered}
$$

We complete the proof by expressing $x(b, c)$ in terms of the generators:

$$
x(b, c)=\left\{\begin{array}{ll}
b \cdot\left(x_{0}+2 \delta_{0}\right)+c \cdot \delta_{0} & \text { if } m=8 \nu+3 \\
b \cdot\left(x_{0}+\left(2+2^{2 \nu+1}\right) \cdot \delta_{0}\right)+\left(c-2^{2 \nu+1} \cdot b\right) \cdot \delta_{0} & \text { if } m=8 \nu+7
\end{array}\right\} .
$$

Let $M=M\left(Q_{2}, k \cdot \tau\right)$ and let $s=\alpha\left(R P^{m}\right) ; s=\alpha\left(R P^{m}\right) \leq \alpha\left(M\left(\mathbf{Z}_{4}, k \cdot \tau\right)\right) \leq$ $\alpha(M)$ by Lemma 1.1. To show $\alpha(M) \leq s$, we must find nonnegative integers $\{A, B, C\}$ so

$$
(T(M) \oplus 1)^{s+1}=A \cdot \pi\left(\rho_{0}\right) \oplus B \cdot \pi(u) \oplus C \cdot \pi(r(\tau)) \oplus(T(M) \oplus 1)^{s} .
$$

Assume for the remainder of this section either $s$ is even or that the inequalities of Corollary 3.2 hold for $(m, s)$. Let $w_{s}=4^{s-1} \cdot k^{s}=4^{-1} \cdot \operatorname{dim}\left\{(1 \oplus T(M))^{s}\right\}$; so $\operatorname{dim}\left(J^{s}(M)\right)=4 \cdot\left(w_{s+1}-w_{s}\right)$. Since $T(M) \oplus 1=k \cdot \pi(r(\tau))$,

$$
(T(M) \oplus 1)^{s}=\left\{\begin{array}{ll}
w_{s} \cdot(1 \oplus \pi(u)) & \text { if } s \text { is even } \\
w_{s} \cdot \pi(r(\tau)) & \text { if } s \text { is odd }
\end{array}\right\} .
$$

If $m=8 \nu+3$, then $f(m)=4 \nu+2$ and $w_{s}<2^{4 \nu} \leq w_{s+1}$. If $m=8 \nu+7$, then $f(m)=4 \nu+3$ and $w_{s}<2^{4 \nu+1} \leq w_{s+1}$. We need nonnegative integers $\{A, B, C\}$ satisfying $A+3 B+4 C=4\left(w_{s+1}-w_{s}\right)$ so that

\begin{tabular}{|llr|}
\hline$s$ even & $s$ odd \\
\hline$B+w_{s} \equiv 0\left(2^{2 \nu}\right)$ & $w_{s+1} \equiv B\left(2^{2 \nu}\right)$ & $m=8 \nu+3$ \\
$w_{s+1} \equiv C\left(2^{4 \nu}\right)$ & $C+w_{s} \equiv 0\left(2^{4 \nu}\right)$ & \\
\hline$B+w_{s} \equiv 0\left(2^{2 \nu}\right)$ & $w_{s+1} \equiv B\left(2^{2 \nu}\right)$ & $m=8 \nu+7$ \\
$2^{2 \nu+1}\left(B+w_{s}\right)+w_{s+1}$ & $2^{2 \nu+1}\left(B-w_{s+1}\right)$ & \\
$\equiv C\left(2^{4 \nu+3}\right)$ & $\equiv w_{s}+C\left(2^{4 \nu+3}\right)$ \\
\hline
\end{tabular}

Define

\begin{tabular}{|lll|}
\hline$s$ even & $s$ odd \\
\hline$A=B=2^{4 \nu}-w_{s}$ & $A=B=w_{s+1}-2^{4 \nu} \quad m=8 \nu+3$ \\
$C=w_{s+1}-2^{4 \nu}$ & $C=2^{4 \nu}-w_{s}$ \\
\hline$A=2^{4 \nu+1}-w_{s}+4 x 2^{4 \nu+1}+3 y 2^{2 \nu}$ & $A=w_{s+1}-2^{4 \nu+1}-3 \cdot 2^{2 \nu}$ & \\
$B=2^{4 \nu+1}-w_{s}-y 2^{2 \nu}$ & $B=w_{s+1}-2^{4 \nu+1}+2^{2 \nu}$ & \\
$C=w_{s+1}-2^{4 \nu+1}-x 2^{4 \nu+1}$ & $C=2^{4 \nu+1}-w_{s}$ & \\
\hline
\end{tabular}


This satisfies the congruences if $1+x-y \equiv 0 \bmod 4$. We must check the positivity condition. If $s$ is odd, $B \geq 0$, and $C \geq 0$. Corollary 3.2(a) implies $A \geq 0$. If $s$ is even, choose $x$ and $y$ as follows:

$$
(x, y)=\left\{\begin{array}{ll}
(0,1) & \text { if } 2^{4 \nu+1}-w_{s}-2^{2 \nu} \geq 0 \\
(1,-2) & \text { if } w_{s+1}-2^{4 \nu+1}-2^{4 \nu+1} \geq 0
\end{array}\right\} .
$$

This yields nonnegative $A, B$, and $C$. If neither inequality holds,

$$
2^{4 \nu}<2^{4 \nu+1}-2^{2 \nu}<w_{s}<2^{4 \nu+1} \leq w_{s+1} \leq 2^{4 \nu+2} .
$$

This implies $m+1=w_{s+1} / w_{s}<4$ which is false. Consequently $\alpha\left(S^{4 k-1} / Q_{2}\right)=$ $\alpha\left(S^{4 k-1} / \mathbf{Z}_{4}\right)=\alpha\left(R P^{4 k-1}\right)$.

We clear the previous notation and redefine $w_{s}$ and $u$. Let $M=M\left(\mathbf{Z}_{4}, \tau\right)$ have dimension $m=4 \nu+1$ where $\tau=(2 \nu+1) r\left(\rho_{1}\right)$. Let

$$
w_{s}=\operatorname{dim}\left\{(T(M) \oplus 1)^{s}\right\} / 2 .
$$

Since $f(m)=2 \nu+1, w_{s}<2^{2 \nu} \leq w_{s+1}$. Let $u=\left[\pi\left\{r\left(\rho_{1}\right)-2 \rho_{0}\right\}\right], v=$ $\left[\pi\left\{\rho_{2}-\rho_{0}\right\}\right]$, and $x(b, c)=b \cdot u+c \cdot v$.

Lemma 3.4. $x(b, c)=0$ if and only if $b-2^{\nu} \cdot c \equiv 0 \bmod 2^{2 \nu+1}$ and $c \equiv$ $0 \bmod 2^{\nu}$.

Proof. We use the calculation $\tilde{K} O(M)=\mathbf{Z}_{2^{2 \nu+1}} \oplus \mathbf{Z}_{2^{\nu}}$ of Kobayashi et al. [7, Theorem B]. The generators are $\left\{u, v+2^{\nu} \cdot u\right\}$. We decompose $x(b, c)=$ $\left(b-2^{\nu} c\right) \cdot u+c \cdot\left(v+2^{\nu} u\right)$.

To show $\alpha(M)=\alpha\left(R P^{m}\right)$, we must find nonnegative integers $\{A, B, C\}$ satisfying

$$
(T(M) \oplus 1)^{s+1}=A \cdot \pi\left(\rho_{0}\right)+B \cdot \pi\left(r\left(\rho_{1}\right)\right)+C \cdot \pi\left(\rho_{2}\right)+(T(M) \oplus 1)^{s} .
$$

Since

$$
(T(M) \oplus 1)^{s}=\left\{\begin{array}{ll}
w_{s} \pi\left(\rho_{0}+\rho_{2}\right) & \text { if } s \text { is even } \\
w_{s} \pi\left(r\left(\rho_{1}\right)\right) & \text { if } s \text { is odd }
\end{array}\right\}
$$

we need $A+2 B+C=2\left(w_{s+1}-w_{s}\right)$ so that

\begin{tabular}{|ll|}
\hline$s$ even & $s$ odd \\
\hline$C+w_{s} \equiv 0\left(2^{\nu}\right)$ & $w_{s+1} \equiv C\left(2^{\nu}\right)$ \\
$B-w_{s+1} \equiv 2^{\nu}\left(C+w_{s}\right)\left(2^{2 \nu+1}\right)$ & $B+w_{s} \equiv 2^{\nu}\left(C-w_{s+1}\right)\left(2^{2 \nu+1}\right)$ \\
\hline
\end{tabular}

Define

\begin{tabular}{|ll|}
\hline$s$ even & $s$ odd \\
\hline$A=2^{2 \nu}-w_{s}+y 2^{\nu}+x 2^{2 \nu+1}$ & $A=w_{s+1}-2^{2 \nu}-2^{\nu}$ \\
$B=w_{s+1}-2^{2 \nu}-x 2^{2 \nu}$ & $B=2^{2 \nu}-w_{s}$ \\
$C=2^{2 \nu}-w_{s}-y 2^{\nu}$ & $C=w_{s+1}-2^{2 \nu}+2^{\nu}$ \\
\hline
\end{tabular}


This satisfies the congruences if $1+x-y \equiv 0 \bmod 2$. If $s$ is odd, then $A, B$, and $C$ are nonnegative by Corollary 3.2(b). If $s$ is even, choose

$$
(x, y)=\left\{\begin{array}{ll}
(0,1) & \text { if } 2^{2 \nu}-w_{s}-2^{\nu} \geq 0 \\
(1,0) & \text { if } w_{s+1}-2^{2 \nu}-2^{2 \nu} \geq 0
\end{array}\right\} .
$$

This yields nonnegative $A, B$, and $C$. If neither condition holds, then

$$
2^{2 \nu-1} \leq 2^{2 \nu}-2^{\nu}<w_{s}<2^{2 \nu} \leq w_{s+1} \leq 2^{2 \nu+1}
$$

This implies $m+1=w_{s+1} / w_{s}<4$ which is false. This completes the proof of Theorem $0.1(\mathrm{e})$.

\section{ODD PRIMES}

This section is devoted to the proof of Theorem $0.1(\mathrm{f})$. Let $n=p^{q}$ be an odd prime power. Recall $\sigma_{1} \geq \sigma_{2}$ if $\sigma_{1}-\sigma_{2}$ is a representation and not just a virtual representation; $c$ and $r$ preserve this partial order. If $\sigma \in R O\left(\mathbf{Z}_{n}\right)$, then $\sigma \geq 0$ if and only if $c(\sigma) \geq 0$. If $V$ and $W$ are vector bundles, define a partial order on the set of vector bundles by $V \geq W$ if there exists $\sigma \geq 0$ so $V=W \oplus \pi(\sigma)$. Let $\delta=\rho_{0}+\cdots+\rho_{n-1}$ be the right regular representation; $\delta \cdot \sigma=\operatorname{dim}(\sigma) \cdot \delta$ for any $\sigma \in R U\left(\mathbf{Z}_{n}\right)$. Let

$$
\left(\rho_{1}+\rho_{-1}\right)^{s}=\sum_{0 \leq \nu<n} a_{\nu}(s, n) \cdot \rho_{\nu} .
$$

Lemma 4.1. Let $n=p^{q}$ and let $M=M\left(\mathbf{Z}_{n}, \tau\right)$ have dimension $m=2 k-1$.

(a) If $\left(\tau+\tau^{*}\right)^{s+1} \geq\left(\tau+\tau^{*}\right)^{s}$, then $\alpha(M) \leq s$.

(b) If $(a, n)=1$, then $\left(\rho_{a}+\rho_{-a}\right)^{n-1} \geq \delta$.

(c) If $1 \leq s \leq n-2, \exists \nu$ so $a_{\nu}(s, n)=0$ and $a_{\nu}(s-1, n) \neq 0$.

(d) Let $c_{\nu} \geq 0$ and $d_{\nu} \geq 0$ with $\sum_{\nu} c_{\nu}=\sum_{\nu} d_{\nu}>m$. Let $x=\pi\left(\sum_{\nu} c_{\nu} \cdot \rho_{\nu}\right)$ and $y=\pi\left(\sum_{\nu} d_{\nu} \cdot \rho_{\nu}\right)$.

(i) If $x=y$, then $c_{\nu} \equiv d_{\nu} \bmod p^{[(k-1) /(n-1)]} \forall \nu$.

(ii) If $c_{\nu} \equiv d_{\nu} \bmod p^{[(k-1) /(p-1)]+q} \forall \nu$, then $x=y$.

Proof. If $c(r(\tau))^{s+1} \geq c(r(\tau))^{s}$, then $s \geq 1$ since $\rho_{0}$ does not appear in $\tau$. Furthermore $r(\tau)^{s+1}=r(\tau)^{s}+\sigma$ so $(T(M) \oplus 1)^{s+1}=(T(M) \oplus 1)^{s} \oplus \pi(\sigma)$ so $J^{s}(M)=\pi(\sigma)$ which proves (a). Expand $\left(\rho_{a}+\rho_{-a}\right)^{s}=\sum_{0 \leq i \leq s}\left(\begin{array}{l}s \\ i\end{array}\right) \cdot \rho_{2 i a-a s}$. If $s=n-1$, since $2 a$ is coprime to $n$, the subscripts $\{-a s,-a s+2 a,-a s+$ $4 a, \ldots, a s\}$ are distinct $\bmod n$; the pigeon-hole principle shows each subscript appears in this set which proves (b). To prove (c), we may suppose $a=1$. $a_{\nu}(s, n)=\sum_{\nu \equiv 2 i-s}\left(\begin{array}{l}s \\ i\end{array}\right)$. If $s<n-1$, there is at most one term in this sum for each $\nu$. If $s$ is odd, then $a_{0}(s, n)=0$ and $a_{0}(s-1, n) \neq 0$; if $s$ is even, $a_{1}(s, n)=0$ and $a_{1}(s-1, n) \neq 0$. This proves $(\mathrm{c})$. Decompose $\widetilde{K} U(M)$ into cyclic summands $\bigoplus_{1 \leq i \leq n-1} \mathbf{Z}_{t(i, k)}$ of orders $t(i, k)=p^{s(i, k)}$. The $s(i, k)$ have been computed by Kobayashi et al. [6, Theorem 1.7] and N. Mahammed [8, Theorem 3] and in particular

$$
[(k-1) /(p-1)]+q \geq s(i, k) \geq[(k-1) /(n-1)] .
$$


To prove Theorem $0.1(\mathrm{f})$, we must show that if $k$ is large with respect to $n$, then

(i) $\alpha(M) \leq n-2$,

(ii) $\exists \tau$ so $\alpha(M)=1$,

(iii) if $\tau=k \cdot \rho_{1}$, then $\alpha(M)=n-2$.

To prove (i), expand $\tau=\sum_{(\nu, p)=1} c_{\nu} \cdot \rho_{\nu}$ and choose $a$ so $c_{a}$ is maximal. Since $c_{a} \geq k / n, c_{a}^{n-1} \geq(k / n)^{n-1} \geq(2 k)^{n-2}$ if $k$ is large. Therefore

$$
\begin{aligned}
\left(\tau+\tau^{*}\right)^{n-1} & \geq c_{a}^{n-1} \cdot\left(\rho_{a}+\rho_{-a}\right)^{n-1} \geq(k / n)^{n-1} \cdot \delta \\
& \geq(2 k)^{n-2} \cdot \delta \geq\left(\tau+\tau^{*}\right)^{n-2} .
\end{aligned}
$$

This proves (i). To prove (ii), let $\tau_{0}=\sum_{(i, n)=1} \rho_{i} ; \operatorname{dim}\left(\tau_{0}\right)<n$. If $k>n^{2}$, let $k=k_{0}+k_{1} \cdot \operatorname{dim}\left(\tau_{0}\right)$ for $k_{0}<n$ and $k_{1}>n$. $\tau_{0}$ is self-dual and $\tau_{0}^{2}=$ $\sum_{(i, n)=1,(j, n)=1} \rho_{i+j}$. Since $p \geq 3$, either $\nu-1$ or $\nu-2$ will be coprime to $p$ for any $\nu$ so any congruence class $\bmod p$ is the sum of two coprime congruence classes; this shows $\tau_{0}^{2} \geq \delta$. Let $\tau=k_{0} \cdot \rho_{1}+k_{1} \cdot \tau_{0}$. Then

$$
\left(\tau+\tau^{*}\right)^{2} \geq k_{1}^{2} \cdot\left(\tau_{0}+\tau_{0}^{*}\right)^{2} \geq 4 k_{1}^{2} \cdot \delta \geq 2 k_{0} \cdot \delta+2 k_{1} n \cdot \delta \geq\left(\tau+\tau^{*}\right) .
$$

If $M=M\left(\mathbf{Z}_{n}, \tau\right)$, then $\alpha(M) \leq 1$. Since $\alpha(M) \geq 1$ for $m \geq 8$, this proves (ii). To prove (iii), let $\tau=k \cdot \rho_{1}$ and let $M=M\left(\bar{Z}_{n}, \tau\right)$. Since $\alpha(M) \leq n-2$ by (i), we show $\alpha(M)=n-2$ by showing $J^{n-3}(M)$ is not flat. Suppose the contrary. Choose $\sigma$ so $(T(M) \oplus 1)^{n-2}=(T(M) \oplus 1)^{n-3} \oplus \pi(\sigma)$. Let $c(\sigma)=\sum_{\nu} c_{\nu} \cdot \rho_{\nu}$ and complexify. Then

$$
\pi\left(\sum_{\nu} a_{\nu}(n-2, n) \cdot \rho_{\nu}\right)=\pi\left(\sum_{\nu}\left(a_{\nu}(n-3, n)+c_{\nu}\right) \cdot \rho_{\nu}\right) .
$$

By Lemma 4.1(d), $a_{\nu}(n-2, n) \equiv a_{\nu}(n-3, n)+c_{\nu} \bmod p^{[(k-1) /(n-1)]}$. Choose $\nu$ so $a_{\nu}(n-2, n)=0$ and $a_{\nu}(n-3, n) \neq 0$. Then $a_{\nu}(n-3, n)+c_{\nu} \equiv$ $0 \bmod p^{[(k-1) /(n-1)]}$ implies $a_{\nu}(n-3, n)+c_{\nu} \geq p^{[(k-1) /(n-1)]}$. Therefore

$$
2^{n-3}+(2 k)^{n-2} \geq a_{\nu}(n-3, n)+c_{\nu} \geq p^{[(k-1) /(n-2)]} .
$$

The left-hand side of the inequality grows polynomially in $k$; the right-hand side of the inequality grows exponentially in $k$. If $k$ is large, this fails; this completes the proof of Theorem $0.1(\mathrm{f})$.

\section{The Classical Lens SPACES}

In Theorem 0.1(f), we showed $\lim _{k \rightarrow \infty} \alpha\left(M\left(\mathbf{Z}_{n}, k \cdot \rho_{1}\right)\right)=n-2$. In general, $n-2$ is not an upper bound. We conclude with a description of the behavior of the alpha invariant for the classical lens spaces if $n=31$; the behavior for other $n$ is similar. 
TABLE 2

\begin{tabular}{|cccc|}
\hline$s$ & $k$ & $s$ & $k$ \\
\hline 51 & {$[4039,4946]$} & 39 & {$[91972,214862]$} \\
49 & {$[4947,7848]$} & 37 & {$[214863,583985]$} \\
47 & {$[7.849,13091]$} & 35 & {$[583986,1961013]$} \\
45 & {$[13092,23183]$} & 33 & {$[1961014,9107278]$} \\
43 & {$[23184,44145]$} & 31 & {$[9107279,77558759]$} \\
41 & {$[44146,91971]$} & 29 & {$[77558760, \infty)$} \\
\hline
\end{tabular}

Theorem 5.1. Let $M_{k}=M\left(\mathrm{Z}_{31}, k \cdot \rho_{1}\right)$.

(a) $\alpha\left(M_{k}\right) \leq 51 \forall k$.

(b) If $k \geq 4039$, then $s(k)=\alpha\left(M_{k}\right)$ is odd and is given by Table 2 .

Proof. As in Lemma 4.1, expand $\left(\rho_{1}+\rho_{-1}\right)^{s}=\sum_{0 \leq \nu<31} a_{\nu}(s) \cdot \rho_{\nu}$ with $a_{\nu}(s)>$ $0 \forall \nu$ if $s \geq 29$. Define the critical ratio by

$$
\operatorname{crit}(s)=\left\{\begin{array}{ll}
\max _{\nu} a_{\nu}(s) / a_{\nu}(s+1) & \text { if } s \geq 29 \\
\infty & \text { otherwise }
\end{array}\right\} .
$$

In addition to the critical ratio, we need to measure the congruences appearing in the $K$-theory groups. Define

$$
\begin{aligned}
f(s, x) & =(s+1) \cdot \ln (2 x)-\{x / 30-1\} \cdot \ln (31) \\
g(s, x) & =(s+1) \cdot \ln (2 x)-\{x / 30+2\} \cdot \ln (31)-\ln (2) \\
& =f(s, x)-3 \cdot \ln (31)-\ln (2) .
\end{aligned}
$$

Let $x_{0}(s)=30 \cdot(s+1) / \ln (31)$.

Lemma 5.2. Let $M=M\left(\mathrm{Z}_{31}, k \cdot \rho_{1}\right)$.

(a) If $k \geq \operatorname{crit}(s)$, then $\alpha(M) \leq s$.

(b) If $g(s, k)>0$, then $\alpha(M) \leq s$.

(c) If $k<\operatorname{crit}(s)$ and if $\alpha(M) \leq s$, then $f(s, k) \geq 0$.

(d) $f$ and $g$ are increasing on $\left[1, x_{0}(s)\right)$ and decreasing on $\left(x_{0}(s), \infty\right)$.

Proof. We use Lemma 4.1. Let $\tau=k \cdot \rho_{1}$. Then

$$
\begin{aligned}
\left(\tau+\tau^{*}\right)^{s+1} & =k^{s+1} \cdot \sum_{\nu} a_{\nu}(s+1) \rho_{\nu}, \\
\left(\tau+\tau^{*}\right)^{s} & =k^{s} \cdot \sum_{\nu} a_{\nu}(s) \rho_{\nu} .
\end{aligned}
$$


If $k \geq \operatorname{crit}(s)$, then $k \cdot a_{\nu}(s+1) \geq a_{\nu}(s) \forall \nu$ so $\left(\tau+\tau^{*}\right)^{s+1} \geq\left(\tau+\tau^{*}\right)^{s}$. Therefore $\alpha(M) \leq s$ which proves (a). If $g(s, k)>0$, then

$$
\begin{gathered}
(2 k)^{s+1} \geq 2 \cdot 31^{k / 30+2}, \\
(2 k)^{s+1}-(2 k)^{s} \geq(2 k)^{s+1} / 2 \geq 31 \cdot 31^{[(k-1) / 30]+1} .
\end{gathered}
$$

We complexify and project to $\widetilde{K} U(M)$. Expand

$$
\gamma\left\{c\left(J^{s}(M)\right)\right\}=\sum_{0<\nu \leq 15} c_{\nu} \cdot\left[\pi\left(\rho_{\nu}+\rho_{-\nu}-2 \rho_{0}\right)\right]
$$

By Lemma $4.1(\mathrm{~d}, \mathrm{ii})$ we may assume $0 \leq c_{\nu} \leq 31^{[(k-1) / 30]+1}$. Let

$$
\begin{aligned}
c_{0} & =\operatorname{dim} J^{s}(M)-2 \cdot \sum_{0<\nu \leq 15} c_{\nu} \\
& \geq(2 k)^{s+1}-(2 k)^{s}-30 \cdot 31^{[(k-1) / 30]+1} \geq 0 .
\end{aligned}
$$

Let $\sigma=\sum_{1 \leq \nu \leq 15} c_{\nu} \cdot r\left(\rho_{\nu}\right)+c_{0} \cdot \rho_{0}$. Then $J^{s}(M)=\pi(\sigma)$ as $c$ is injective. This shows $\alpha(M) \leq s$ and proves (b). Suppose $k<\operatorname{crit}(s)$ and that $J^{s}(M)$ has a flat structure. Let $c\left(J^{s}(M)\right)=\pi(\sigma)$ for $\sigma=\sum_{\nu} c_{\nu} \cdot \rho_{\nu}$. Then $c(T(M) \oplus 1)^{s+1}=$ $\pi(\sigma) \oplus c(T(M) \oplus 1)^{s}$ so

$$
k^{s+1} \cdot a_{\nu}(s+1) \equiv k^{s} \cdot a_{\nu}(s)+c_{\nu} \bmod 31^{[(k-1) / 30]} \forall \nu .
$$

Since $k<\operatorname{crit}(s)$, we may choose $\mu$ so $k^{s+1} \cdot a_{\mu}(s+1)<k^{s} \cdot a_{\mu}(s)$. This implies

$$
\begin{gathered}
k^{s} \cdot a_{\mu}(s)+c_{\mu}-k^{s+1} \cdot a_{\mu}(s+1)>0, \\
k^{s} \cdot a_{\mu}(s)+c_{\mu}-k^{s+1} \cdot a_{\mu}(s+1) \geq 31^{[(k-1) / 30]}, \\
k^{s} \cdot a_{\mu}(s)+c_{\mu} \geq 31^{[(k-1) / 30]} .
\end{gathered}
$$

As $a_{\nu}(s)$ and $c_{\nu}$ are nonnegative for all $\nu$, we sum to see

$$
\begin{gathered}
(2 k)^{s+1}=k^{s} \cdot \sum_{\nu} a_{\nu}(s)+\sum_{\nu} c_{\nu} \geq 31^{[(k-1) / 30]}, \\
(s+1) \cdot \ln (2 k) \geq[(k-1) / 30] \cdot \ln (31) \geq\{k / 30-1\} \cdot \ln (31) .
\end{gathered}
$$

This shows $f(s, k) \geq 0$ which proves $(\mathrm{c})$. Finally

$$
\begin{gathered}
\partial g / \partial x(s, x)=\partial f / \partial x(s, x)=(s+1) / x-\ln (31) / 30 \\
\partial^{2} f / \partial x^{2}(s, x)=-(s+1) / x^{2}<0 .
\end{gathered}
$$

The critical point is at $x_{0}=30 \cdot(s+1) / \ln (31)$.

The approximate values of $\operatorname{crit}(s)$ are given in Table 3. 
TABLE 3

\begin{tabular}{|crrr|}
\hline$s$ & \multicolumn{1}{c}{$\operatorname{crit}(s)$} & \multicolumn{1}{c}{$s$} & \multicolumn{1}{c|}{$\operatorname{crit}(s)$} \\
\hline 29,30 & 77558760.0 & 41,42 & 44145.2 \\
31,32 & 9107278.6 & 43,44 & 23183.3 \\
33,34 & 1961013.6 & 45,46 & 13091.5 \\
35,36 & 583985.5 & 47,48 & 7848.2 \\
37,38 & 214862.2 & 49,50 & 4946.5 \\
39,40 & 91971.5 & 51 & 3253.04 \\
\hline
\end{tabular}

TABLE 4

\begin{tabular}{|rrrrr|}
\hline$s$ & \multicolumn{1}{c}{$x$} & $f(s, x)$ & $g(s, x)$ & \multicolumn{1}{c|}{$x_{0}(s)$} \\
\hline 50 & 4038 & .05 & -10.95 & 445.55 \\
50 & 4039 & -.05 & -11.05 & 445.55 \\
51 & 1 & 39.36 & 28.37 & 454.28 \\
51 & 3253 & 87.66 & 76.67 & 454.28 \\
51 & 4946 & -84.34 & -95.34 & 454.28 \\
\hline
\end{tabular}

The critical ratio for 29 and 30 is exact. We also computed approximations to $f, g$, and $x_{0}$ in Table 4 .

Since $g(51,1)>0$ and $g(51,3253)>0, g(51, x)>0$ for $x \in[1,3253]$. By Lemma 5.2(b), $\alpha\left(M_{k}\right) \leq 51$. If $k>3253$, then $k>\operatorname{crit}(51)$ so $\alpha\left(M_{k}\right) \leq$ 51 by Lemma 5.2(a). This shows $\alpha\left(M_{k}\right) \leq 51 \forall k$. Since $x_{0}(50)<4039$ and $f(50,4039)<0, f(50, x)$ is negative on $[4039, \infty)$. If $k \in[4039,4946]$, then $k<\operatorname{crit}(50)$ so $\alpha\left(M_{k}\right)>50$ by Lemma $5.2(\mathrm{c})$. This shows $\alpha\left(M_{k}\right)=51$ for $k \in[4039,4946]$. Let $k \geq 4947$ and $s \in[29,49]$. Both $x_{0}(s)$ and $f(s, k)$ are increasing in $s$. Since $x_{0}(s) \leq x_{0}(51)=469.4, f(s, k)$ is decreasing in $k$. Thus $f(s, k) \leq f(51, k) \leq f(51,4946)<0$. By Lemma 5.2(a, c),

(i) if $k \geq \operatorname{crit}(s)$, then $\alpha(M) \leq s$ and

(ii) if $k<\operatorname{crit}(s)$, then $\alpha(M)>s$.

If $k \in[4947,7848]$, then $\operatorname{crit}(49) \leq k<\operatorname{crit}(48)$ so $48<\alpha\left(M_{k}\right) \leq 49$; the computation of $\alpha$ for the remaining ranges is the same.

\section{BIBLIOGRAPHY}

1. R. Bott and J. Milnor, On the parallelizability of spheres, Bull. Amer. Math. Soc. 64 (1958), 87-89.

2. K. Fujii, On the KO-ring of $S^{4 n+3} / H_{m}$, Hiroshima Math. J. 4 (1974), 459-475.

3. P. Gilkey, The eta invariant and the $K$-theory of odd dimensional spherical space forms, Invent. Math. 76 (1984), 421-453.

4. _ The eta invariant and KO of lens spaces, Math. Z. 194 (1987), 309-31020.

5. P. Gilkey and M. Karoubi, K-theory for spherical space forms, Topology Appl. 25 (1987), 179-184.

6. T. Kobayashi, S. Murakami and M. Sugawara, Note on J-groups of lens spaces, Hiroshima Math. J. 7 (1977), 387-409. 
7. T. Kobayashi and M. Sugawara, $K_{\Lambda}$-rings of lens spaces $L^{n}(4)$, Hiroshima Math. J. 1 (1971), 253-271.

8. N. Mahammed, K-théorie des espaces lenticulaires, C. R. Acad. Sci. Paris 272 (1971), 13631365.

9. T. N. Shorey and R. Tijdeman, New applications of diophantine approximations to diophantine equations, Math. Scand. 39 (1976), 5-18.

10. J. Turk, On the difference between perfect powers, Acta Arith. 45 (1986), 289-307.

11. J. Wolf, Spaces of constant curvature, 5th ed., Publish or Perish, Wilmington, Del., 1984.

Department of Mathematics, Lewis and Clark College, Portland, Oregon 97219

Department of MAthematics, University of Oregon, Eugene, Oregon 97403

Department of Mathematics, The Wichita State University, Wichita, Kansas 67208 